\title{
An Architecture for Distributed and Flexible Management of High-Layer Protocols and Network Services
}

\author{
Luciano Gaspary $^{1,2}$, Luis F. Balbinot ${ }^{1}$, Roberto Storch ${ }^{1}$, Fabrício Wendt $^{1}$ and Liane Tarouco ${ }^{1}$ \\ ${ }^{1}$ Federal University of Rio Grande do Sul \\ Instituto de Informática \\ Av. Bento Gonçalves, 9500 - Agronomia - CEP 91.591-970 \\ Porto Alegre, Brazil \\ (hades, storch,wendt)@inf.ufrgs.br and liane.tarouco@ufrgs.br \\ ${ }^{2}$ Universidade do Vale do Rio dos Sinos \\ Centro de Ciências Exatas e Tecnológicas \\ Av. Unisinos, 950 - CEP 93.022-000 \\ São Leopoldo, Brazil \\ paschoal@exatas.unisinos.br
}

\begin{abstract}
This paper proposes an architecture for distributed management of high-layer protocols and network services. Based on the IETF Script MIB, the Trace architecture provides mechanisms for the delegation of management tasks to mid-level managers (MLMs), which interact with monitoring and action agents to have them executed. The paper introduces PTSL (Protocol Trace Specification Language), a graphical/textual language created to allow network managers to specify protocol traces. The specifications are used by mid-level managers to program the monitoring agents. Once programmed, these agents start to monitor the occurrence of the traces. The information obtained is analyzed by the mid-level managers, which may ask action agents for the execution of procedures (e.g. Perl scripts), making the automation of several management tasks possible.
\end{abstract}

Keywords: Internet management, network management, network monitoring, scripting.

\section{Introduction}

The use of computer networks to support an increasing number of businesses and critical applications has stimulated the research for new management solutions that maintain not only the physical network infrastructure, but also the protocols and services that flow over it. The popularization of electronic commerce (e-commerce) and the increasing use of this business modality by companies, for instance, implies using the network to exchange critical data from the organization and from its customers. Protocols and services that support these applications are critical and, therefore, need to be carefully monitored and managed.
Not only critical applications require special attention. New protocols are frequently released to the market to support an increasing set of specific functionalities. These protocols are quickly adopted by network users. As a result of this fast proliferation, weakly-tested and even faulty protocols are disseminated to the market. In several cases these anomalies, as well as the miscalculated use of resources, are the cause of network performance degradation and end up unperceived by network managers. These factors make the management of these protocols and services an ever increasing endeavor.

For the network manager to be able to provide assured network high-availability and efficiency, a flexible management environment that can be quick and easily adapted to monitor dynamic scenarios is needed. Besides being flexible, the size of current networks requires this management environment to be distributed, so that the solution can be efficient and scalable.

This paper presents an architecture for distributed and flexible management of high-layer protocols and network services based on programmable agents and is organized as follows. Section 2 describes and compares expressive initiatives related to both high-layer protocol and distributed management. Section 3 presents PTSL, a graphical/textual language created to specify protocol traces. Section 4 introduces the Trace architecture and its components. In Section 5 some cases studies that validate the proposal are presented. Section 6 closes the paper by presenting an evaluation of the proposed architecture, some conclusions and future work.

\section{Related Work}

Many approaches have been proposed to both high-layer protocol management and distributed management. When 
it comes to high-layer protocols management, monitoring is the main topic of research. The ntop [1] is designed for traffic measurement and monitoring, and includes features for per-protocol network traffic characterization and usage. The Remote Network Monitoring Management Information Base Version 2 (RMON-2) [2], created in 1997, provides mechanisms to collect information similar to ntop.

Other recent efforts related to monitoring are the extensible architecture proposed by Malan and Jahanian [3] and the Realtime Traffic Flow Measurement (RTFM), developed by the group with the same name at the IETF [4] and implemented by the NeTraMet tool [5]. The RTFM architecture is based on distributed agents (called meters) that implement the RTFM Meter MIB [6]. These agents are capable of making realtime packet flow measurement and accounting. The MIB allows an SNMP agent to query statistical data, as well as set agent configuration data. Flow specifications are made through a set of rules defined by a language called SRL [7] and determine (a) which flows should be counted, (b) which nodes should be treated as flow origins and (c) which level of detail is desired for each flow.

A demand instigated by the fast proliferation of protocols and applications that flow over today's computer networks is the flexibility of monitoring tools. Many existing tools are not completely prepared to allow the monitoring of new protocols and applications and operate on a fixed set of them. New protocols can only be monitored through firmware updates, as with some RMON-2 probes, or by low-level programming languages, like the architecture proposed by Malan and Jahanian and the ntop tool. Many network managers just end up neglecting these possibilities due to their complexity.

Other solutions, like Tivoli Enterprise [8], are intrusive, due to the fact that they require applications to be developed using specific monitoring procedure calls. This approach is only suitable when monitoring is done within applications developed in-house, but it can not be used to manage proprietary protocols and/or applications (e.g. web browsers and clients). Besides, it is also needed to spend more money on personnel training on how to use the monitoring APIs.

The type and granularity of the collected information are important aspects associated with the monitoring. The RMON2 MIB and the ntop tool collect only statistics like the number of packets sent/received by a host or the number of packets exchanged between two peers, classified accordingly to the protocol used (e.g. HTTP and FTP). Advantages and disadvantages of the RMON2 MIB have been shown by Gaspary et al. in [9]. One of the weaknesses of both approaches is the lack of information related to performance and faults. These difficulties have been discussed by the IETF RMON working group through the Application Performance Measurement MIB (APM MIB) [10].

When it comes to granularity, accounting on the RMON2 MIB is made per host, pairs of hosts and protocol used. In the case of the ntop tool, it is possible to recognize and count packet flows, which are specified by a set of lowlevel rules that are processed by the BSD Packet Filter (BPF). In the RTFM architecture, only predetermined protocol fields can be read from captured packets (only up to the transport layer). Information about high-layer protocols can not be considered due to this limitation. Besides, as it occurs with the ntop tool, the same set of rules is applied to each captured packet, making it impossible to correlate messages from a same flow.

That is also very important to note that many management tools like $[1,3,5]$ are limited to monitoring, leaving reactive and/or proactive management to the human manager when an unexpected network behavior is observed.

As for distributed management, Schoenwaelder et al. present in [11] several approaches and existing technologies for its deployment. Technologies based on the dynamic delegation of management tasks and, in special, the potential of delegation of those tasks through the IETF Script MIB [12] are discussed and commented. By using practical examples, they show how the monitoring of thresholds and services can be delegated to mid-level managers.

The next sections present a new approach for high-layer network protocols and services management. The evaluation of this approach contrasted to other approaches mentioned earlier in this section is presented in Section 6.

\section{Protocol Trace Representation}

This section presents PTSL (Protocol Trace Specification Language), a language for the representation of protocol traces based on the concept of finite state machines (FSM). The language is composed of graphical (Graphical PTSL) and textual (Textual PTSL) notations. These notations are not equivalent. The textual notation allows the complete representation of a trace, including the specification of the FSM and the events that trigger transitions. In turn, the graphical notation covers only a subset of the textual notation, offering the possibility of graphically representing the FSM and only labelling the events that trigger transitions. 


\subsection{Graphical PTSL}

The network manager can create a specification to monitor the whole protocol or just part of it. Interactions between more than one protocol can also be represented. Figure 1 shows two trace examples. On the first case (a), the trace monitors successful transactions to a web server. The second trace (b) does not describe a single protocol; it

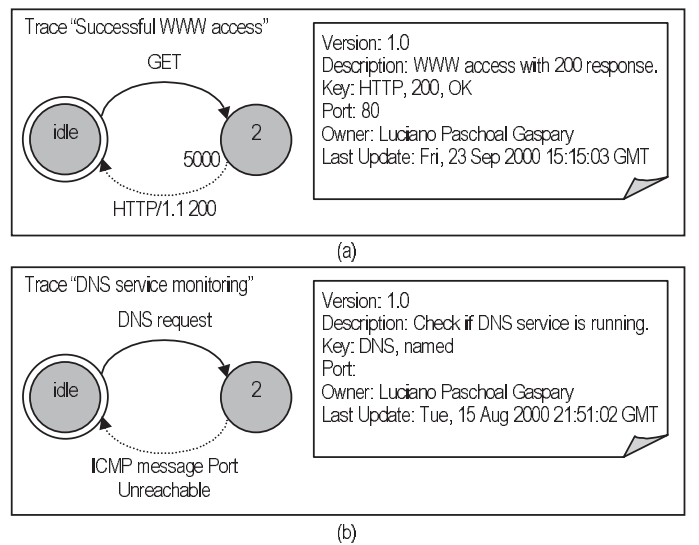

is rather made up of a name resolution request (DNS protocol), followed by an ICMP Port Unreachable message. This trace occurs when the host where the service resides is on, but the named daemon is not running.

Figure 1: Graphical representation of a trace. (a) Successful WWW request. (b) DNS request not replied because named daemon is not executing.

\subsubsection{Representation of states and transitions}

States are represented by circles. From the initial state (idle) other $n$ states can be created, but they must always be reachable through any given transition. The final state is identified by two concentric circles. On both examples (figure 1) the initial and final states are the same. The state transitions are represented by unidirectional arrows. The continuous arrow indicates that the transition is triggered by the client host, while the dotted arrow determines that the transition is triggered by an event coming from the server host. The text associated with a transition is merely a label to the event that triggers it; the full specification can only be made via textual notation.

\subsubsection{Representation of timers}

Transitions, by default, do not have a time limit to be triggered. To associate a timeout with a transition, an explicit value (in milliseconds) must be set. In the example shown in figure $1 \mathrm{a}$, the value 5000 associated to transition HTTP/1.1200 indicates that the transition from state 2 to the initial state has up to five seconds to be triggered.

\subsubsection{Representation of information for cataloguing and version control}

The graphical notation also offers a constructor where information about the trace, which are relevant to cataloguing and version control of specifications, are included (see figure 1). The data stored for a trace are:

Version (the version of the specification), Description (a brief description of the trace), Key (keywords related to the trace), Owner (individual responsible for the trace definition) and Last Update (timestamp of the last update). Besides these data, there is also a Port field, used to indicate the TCP or UDP port of the monitored protocol; this should only be defined when the trace is limited to a single protocol.

\subsection{Textual PTSL}

Figure 2 presents the textual specification of the trace previously shown in figure $1 \mathrm{a}$. All specifications written in Textual PTSL start with the Trace keyword and end with the EndTrace keyword (lines 1 and 30). Catalog and version control information come right after the Trace keyword (lines 2-7). Forthwith, the specification is split into three sections: Messagessection (lines 8-20), Groupssection (not used in this example) and Statessection (lines 21-29). On MessagesSection and Groupssection the events that trigger transitions are defined. The FSM that specifies the trace is defined in Statessection.

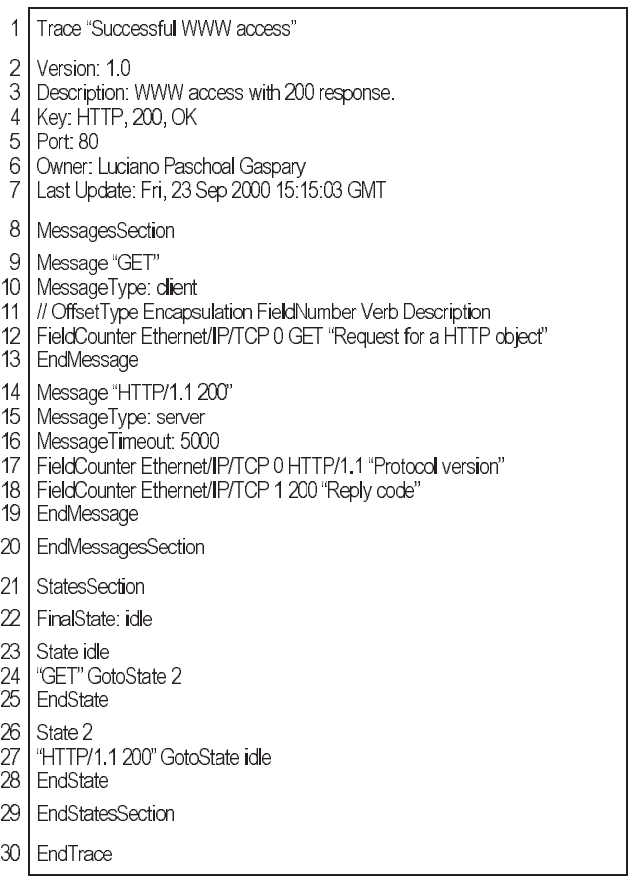

Figure 2: Protocol trace representation using Textual PTSL 


\subsubsection{Representation of messages}

Whenever the fields of a captured packet match the ones specified at a Message for the current state, a transition is triggered in the FSM. The way those fields are specified depends on the type of protocol to be monitored. In the case of variable-length character-based protocols where fields are split by white space characters (HTTP and SMTP, for instance), the identification of a field is made by its position within the message (this is called the FieldCounter strategy). In HTTP/1.1 200, for instance, HTTP/1.1 is at position 0 and 200 is at position 1 . On the other hand, the identification of binary protocols, known by their fixed length fields (e.g. TCP), is determined by a bit offset starting from the beginning of the protocol header; it is also needed to specify the size of the field, in bits (this is the BitCounter strategy).

The trace shown in figure 1a is for a character-based protocol. The GET message specification is shown in figure 2 (lines 9-13). In line 10 the message is defined as being of type client, meaning that the state transition associated with the message will be triggered by the client host. In line 12 the only field to be analyzed is specified. The information necessary to identify it are: fetch strategy (FieldCounter), protocol encapsulation (Ethernet/IP/TCP), field position (0), expected value (GET) and, optionally, a field description. Character-based protocol fields are always identified by this quintuple. The trace reply message HTTP/1.1 200 is shown in lines 14-19. The message type is defined in line 15 as server, i.e., the state transition will be triggered by the server host. In line 16 the MessageTimeout is set to 5000 . Finally, the two fields to be analyzed are defined (lines 17 and 18).

As opposed to the example mentioned above, the trace specified by the messages in figure $1 \mathrm{~b}$ is based on binary protocols, DNS and ICMP. A DNS request will trigger a state change on the FSM from idle to 2 . To recognize a DNS request from the packets flowing over the network two fields must be observed: $\mathrm{QR}$ (when set to 1 indicates a request to the server) and OPCODE (when set to 0 represents a standard query). Field $Q R$ is 16 bits away from the beginning of the header and its size is 1 bit. Field OPCODE starts in the seventeenth bit and occupies 4 bits.

Figure 3 presents the textual specification of a DNS request (lines 1-6). In line 4 the $Q R$ field is defined. The information needed to identify a binary protocol field are: fetch strategy (BitCounter), protocol encapsulation (Ethernet/IP/UDP), field position (16), field length (1), expected value (1) and, optionally, a field description. The information used to identify the OPCODE field are BitCounter, Ethernet/IP/UDP, 17, 4 and 0. The same strategy is used in the definition of ICMP message Port Unreachable in lines 7-11.

\subsubsection{Representation of message groups}

The PTSL language allows the binding of a single transition to multiple distinct events. To do that, the Group constructor must be used within the Groupssection section. The trace presented in figure 1a monitors the occurrence of successful HTTP accesses. However, only accesses with reply code 200 are counted. Accesses with reply codes 201, 202, 203, 204, 205 and 206, that also represent successful operations, could be included into this accounting. For that to be possible, the messages that identify these accesses must be defined (similarly to lines 1419 in figure 2) and grouped (see figure 4). In lines 2-3 all messages that make part of the group are listed. In the graphical representation (figure 1a), the label associated with the transition from state 2 to idle changes from HTTP/1.1200 to HTTP/1.1 20X, which is the name of the new message group (line 1 below).

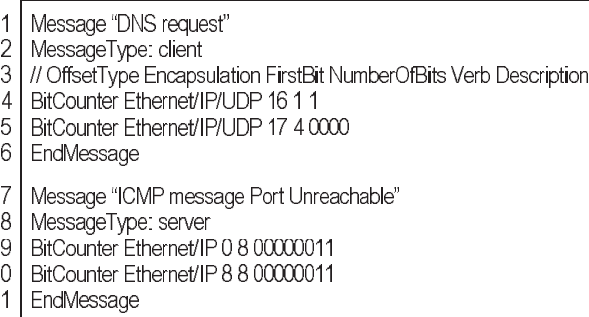

Figure 3: Field identification in binary protocols

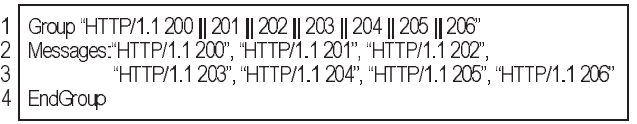

Figure 4: Representation of message groups

\subsubsection{Representation of the FSM}

Lines 21-29 in figure 2 define the textual specification of the state machine shown in figure 1a. The final state is identified just after StatesSection (line 22). The states idle and 2 are defined in lines 23-25 and 26-28. The state specification only lists the events (messages and groupings) that may trigger transitions, indicating, for each one, which is the next state (lines 24 and 27).

\section{The Trace Architecture}

The Trace architecture is an extension of the SNMP centralized management infrastructure. Through a three-tier 
model, it supports the distributed management of highlayer protocols and network services. Figure 5 illustrates the architecture's scheme. Based on the IETF Script MIB [12], it provides mechanisms to allow a management station to delegate management tasks to mid-level managers (MLMs) that, in turn, interact with monitoring and action agents to execute these tasks. PTSL specifications are used by MLMs to program monitoring agents that start sniffing packets flowing on the network and wait for traces to happen. With the information gathered from the monitoring process, the MLMs may launch procedures on action agents (Tcl or Perl scripts), enabling the automation of several management tasks (including reactive and proactive tasks). The architecture also has notification mechanisms (traps) so that agents are able to report asynchronous events to scripts running on MLMs. These MLMs are then able to filter and/or correlate these traps and signal the occurrence of major events to the network management station (NMS). The components of the architecture are presented below.

\subsection{Management Station}

The architecture is made of one or more management stations (managers). If the interfaces between all components are respected, nothing hinders management applications from being developed with different technologies. However, figure 5 suggests a web-based management interface. Through a web browser, the human manager has access to the management environment located in a web server. For convenience, our research group chose the PHP language and the MySQL database to develop this environment. The highlighted modules on the management station may be hosted in the same host where the manager resides. If there is more than one management station, they may share the same environment core.

The most important tasks accomplished by the network manager from a management station are:

- Registration of MLMs and agents: to ease the coordination between the management station, MLMs and agents, the network manager must define who are the MLMs on the network, as well as the agents located (hierarchically) bellow these managers. This binding is important to define management boundaries. When programming a management task, the MLM will only manipulate those agents it is a parent of. The necessary interactions to this registration are presented in figure 5 (numbers 1,2 and 3). This numbering will be used henceforth in this section to illustrate the architecture's data flow.
- Specification of a protocol trace (PTSL script): using the language introduced in Section 3, it is possible to specify a protocol trace. The network manager may specify the trace from scratch or reuse existing traces stored in a repository, derivating a new specification based on previously defined traces (flows 1, 2 and 3 in figure 5). For further use of this trace specification, it must be mapped from the database to a text file and put within the repository (4).

- Specification of an action (Java, Perl or Tcl script): the action scripts do not necessarily have to be specified using the web-based environment facilities. It is possible to upload a script developed in Java, Perl or Tcl to the repository $(1,2$ and 4$)$. It is recommended to exhaustively test these scripts before sending them to a repository. Most Script MIB runtime environments offer debugging capabilities, but some do not.

- Specification of a management task (Java, Perl or Tcl script): by using a wizard the management environment provides, the network manager specifies a management task (flows 1, 2 and 3 in figure 5). When defining the task, the network manager informs the trace to be observed, the identification of the object belonging to the extended RMON2 MIB (explained later), where the observation of the chosen protocol trace will be counted, the polling interval and the actions to be triggered when certain thresholds are reached. These specifications, as usually happens with PTSL specifications, are kept within the database.

- Delegation of a management task: to delegate a task to an MLM, the task must be retrieved from the database (1,2 and 3). Besides, the network manager must choose the mid-level manager, the monitoring agent and the action agent (the latter is not mandatory) that will be responsible for the execution of the task. A corresponding Tcl script is automatically generated and made available at the repository (4). After going through these steps, the execution of the script is delegated to the MLM $(5,6)$ via SNMP (Script MIB).

- Monitoring of a management task: during the execution of a management task, the manager may query the MLM to get intermediate results of the running task (1, 2,5 and 6 ). 


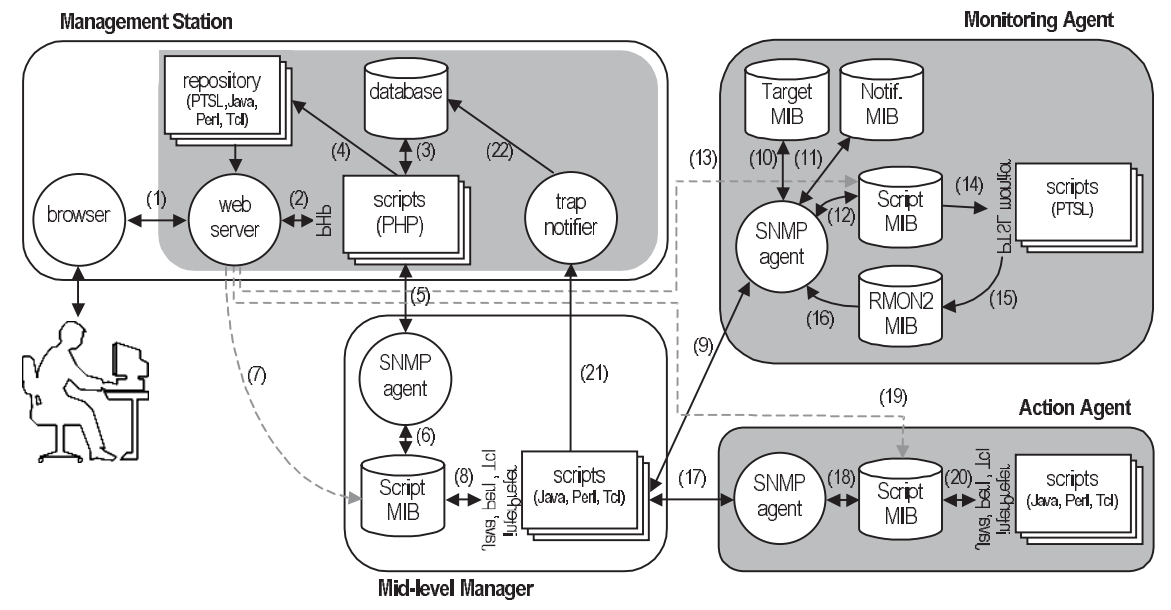

Figure 5: Components of the Trace architecture

- Interruption of a management task: the interruption of a management task requires the removal of all programming made on the monitoring and action agents involved. Only after that it will be possible to terminate the execution of the script (i.e. the management task) at the MLM (1, 2, 5 and 6). It is important to mention that the latest release of the Script MIB provides mechanisms to automatically expire and remove old (possibly forgotten) entries.

- Receiving and viewing traps: the manager may receive traps through a module called Trap Notifier (21). When received, all traps are stored in the database (22). Traps are permanently retrieved by a script (3) that updates the manager's web browser (2 and 1) using the HTTP push technology.

\subsection{Mid-level Manager}

The MLM runs and monitors management tasks delegated by NMSs and reports major events back to these stations. There may be one or more MLMs inside each network. The number of MLMs is determined by the network manager and depends on several factors (e.g. the size and complexity of the network infrastructure or human administrative boundaries).

The delegation of a task to a MLM, as mentioned, is performed by NMSs through SNMP primitives, which are supported by the PHP language (flows 5 and 6 in figure $5)$. When a new entry is created on the Script MIB launch table, the agent automatically downloads the script from the configured URL (7). After this table entry is enabled, the agent is then ready to start running the script (8).

As stated before, the management tasks specified by the network manager are automatically converted to $\mathrm{Tcl}$ scripts in order to be run by MLMs. Although Jasmin [14] (the Script MIB implementation used in our prototype) also supports Java and Perl, we have chosen Tcl because it has inherent network management characteristics and several libraries to support network management operations, besides being flexible and portable. The complexity of scripts run by MLMs is not a critical factor since all specification and delegation of management tasks is made by wizards, even though Tcl scripts can be easily written and understood by those not familiar with the language.

Figure 6 presents a sample script used to monitor the occurrence of a trace. In lines 8-11 and 12-16 the monitoring and action agents are programmed, respectively. The monitoring agent is, in line 17 , asked to start observing the network for the occurrence of the trace just programmed. Then, the MLM polls it every 120 second (line 28) to get information (lines 3 and 20) and checks whether the trace has been counted or not (line 22). If the trace has been observed three times within an interval, another script is launched at the action agent (line 23), to run a management procedure. Intermediate and final results are generated by the script (lines 28 and 29) and made available in the Script MIB.

The script running at the MLM can configure which traps it wishes to receive by using the Target and Notification MIBs installed on monitoring and action agents. On the Target MIB, the MLM sets its IP address and UDP port number to where traps are sent $(9,10)$ (this port number must be unique among all scripts running at the MLM). The Notification MIB allows the script to set which traps it wishes to receive (these are filtered at the notifier) (9, 11) [15]. If the script implements a trap handler, it can run a procedure whenever a trap arrives. Traps can be correlated and a more valuable notification may be sent to the 
NMS (21). This configuration of trap sinks eases the implementation of monitoring and action scripts, since they do not have to care about which are their trap sinks and which credentials should be used to send them.

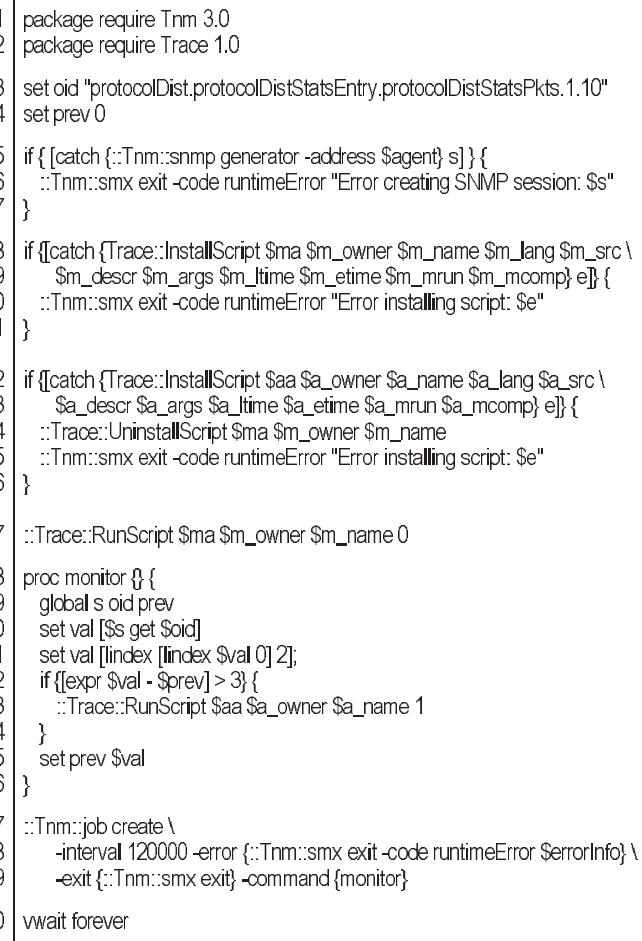

Figure 6: Sample script run by MLMs

It can be noted that the communication between MLMs and monitoring or legacy SNMP agents (handled by the Tcl scripts) is made through SNMP primitives provided by Tcl through the Scotty package. The same happens between MLMs and the management station when traps are sent. The programming of the Script MIB on the monitoring $(9,12)$ and action agents $(17,18)$ is made with the aid of a specially developed Tcl package (line 2 in 6), called Trace.

\subsection{Monitoring Agent}

The monitoring agents count the occurrence of traces on the network segment where they are located. They are said extensible because the monitoring traces can be dynamically configured. This flexibility is possible through the PTSL language. These agents read PTSL files, organize some data structures in memory and start the monitoring process.

The configuration of which traces should be monitored at a given moment is made by the MLM through the Script MIB (9 and 12). On the script run by the MLM (figure 6) it is possible to see how the monitoring agent is programmed (lines 8-11). One of the parameters passed is the URL of the script (PTSL specification) that will be run. When the MLM requests the installation and execution of a script, it is retrieved from the repository via HTTP (13) and executed (14).

Actually, the PTSL is not executable. The semantics associated to line 17 in figure 6 makes the monitoring agent start monitoring a new trace. In an analogous way, the interruption of a script on the Script MIB means programming the monitoring agent so that it ceases monitoring the trace defined by the script.

Every time a trace is observed between any pair of peers, data are stored in a MIB similar to RMON2 [2, 9] (15). One of the differences between this MIB and the RMON2 MIB is that the protocolDir group, which indicates which protocol encapsulations the agent is capable to monitor, now allows protocol traces to be indexed.

The alMatrix group from the RMON2 MIB stores statistical data about the trace when it is observed between each pair of peers. Table 1 illustrates the contents of the alMatrixSD table. It accounts the number os packets/octets between each pair of peers (client/server).

\begin{tabular}{c|c|c|c|c}
\hline Scurce & Destination & Frotccol & Packets & Octets \\
\hline \hline 172.16 .108 .1 & 172.16 .108 .2 & DNS service moritcring & 4 & $4.35 C$ \\
172.16 .108 .32 & 172.16 .108 .2 & DNS service moritcring & 8 & $7.3 C \mathrm{CC}$ \\
172.16 .108 .1 & 172.16 .108 .254 & SLccessful WWW access & 254 & 1.202 .126 \\
$125.120 .10 .1 \mathrm{CC}$ & 172.16 .108 .254 & Unsuccessful TCF ccnnection attempt & 20 & $3.2 \mathrm{CC}$ \\
\hline
\end{tabular}

Table 1: Information from the alMatrixSD table

One disadvantage of the RMON2 MIB is that it does not have the capability to generate information about performance. For this reason, our group is currently evaluating the possibility of using an extension to the RMON2 MIB, the Application Performance Measurement MIB [10]. Table 2 presents the type of information stored by this MIB. The first line indicates that the Successful WWW access trace was observed 127 times between hosts 172.16.108.1 and 172.16.108.254. The number of traces that did not complete with success was 232 and the mean response time for successful observations was 6

\begin{tabular}{c|c|c|c|c|c}
\hline Client & Server & Prctccd & Success. & Unsuccess. & Responsiv. \\
\hline \hline 172.16 .108 .1 & $172.16 .1 C 8.254$ & Successful WWW access & 127 & 232 & $6 \mathrm{sec}$. \\
172.16 .108 .1 & $2 C 0.248 .252 .1$ & Successful WWW access & 232 & 112 & $17 \mathrm{sec}$. \\
$10.1 \mathrm{C} .135 .125$ & $2 C 0.248 .252 .1$ & SYN. Flocd & 10.234 & 56 & $3 \mathrm{sec}$. \\
\hline
\end{tabular}

seconds.

Table 2: MIB with performance information 


\subsection{Action Agent}

Through monitoring agents, MLMs are able to evaluate whether a trace has occurred or not. Traces may represent network service failures, intrusion attempts, service performance degradation, and other problems. In this context, the action agents are responsible for the execution of reactive (and potentially proactive) management procedures created to autonomously handle these problems. Let's take, for instance, the DNS service monitoring. When a mid-level manager detects that the service is not running (through the monitoring loop), it can ask an action agent (located on the same host of the service) to run a script to restart the service such as the one shown in figure 7 .

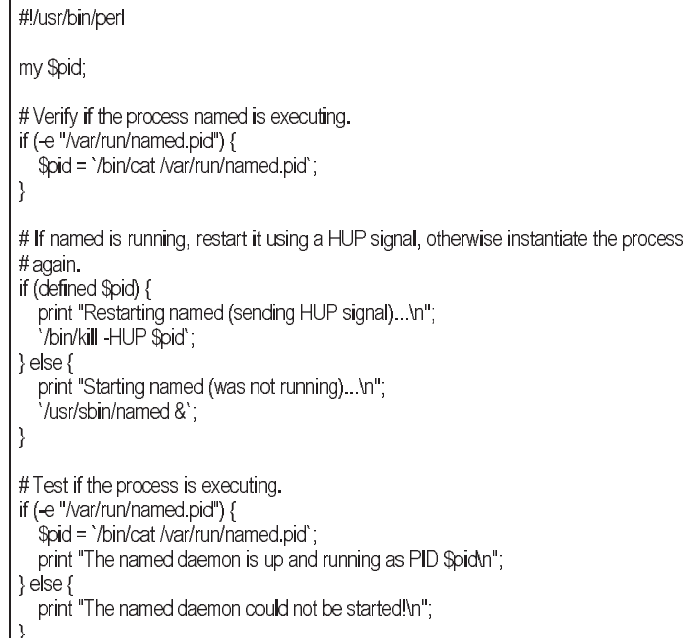

Figure 7: Perl script to restart the daemon named

The communication between MLMs and action agents is made through the Script MIB (see flows 17 and 18 in figure 5). Once the Script MIB is programmed to run an action script, it is retrieved via HTTP from the repository (19) and then executed (20). The script illustrated in figure 7 was written using Perl language. Although most network managers are more familiar with Perl, this language is not mandatory; Java and Tcl can also be used.

\section{Case Studies}

The Trace architecture was designed to allow the management of all functional areas (FCAPS). Our group explored, through a case study, the characteristics of the architecture to validate its applicability on the management of high-layer protocols and network services. Figure 8 shows a real management scenario, composed of three domains. The organization of these domains is a task that the network manager must handle to efficiently use the archi- tecture. This task is accomplished at the management environment when the MLMs and the agents are configured.

Domain 1 is composed of equipment and services related to the organization's Internet access (dark gray in the figure). The router acts as a gateway for three distinct networks: the Internet, the internal network demilitarized zone (where the web and DNS servers are) and the protected intranet (with an Intranet server). There are two monitoring agents $\left(M_{1}\right.$ and $\left.M_{2}\right)$ installed on dedicated monitoring stations and one action agent installed on the same host where the DNS server resides. Based on this scenario, our work group defined some management tasks.

\subsection{Monitoring of the DNS Service Availability}

This management task consists in observing the availability of the name resolution service. Through the Tcl script presented in Figure 6, the mid-level manager responsible for domain 1 programs the monitoring agent $M_{1}$, which is located on the same segment as the DNS server, in order to watch for the occurrence of the trace DNS service monitoring (figure 1b). If this trace is observed at least three times during a polling interval, the mid-level manager will request the action agent $\mathrm{A}$ to launch the script that restarts the service (figure 7).

\subsection{Accounting Accesses to the Web Server}

This task consists in measuring the amount of accesses to the web server, not only successful accesses, but also access failures and unauthorized access attempts. These information allow the manager to, for instance, (a) know the most critical access times and upgrade or configure the server to support more simultaneous connections, (b) count the occurrence of problems with HTTP clients and minimize the problem by revising the pages and (c) reconfigure the web server and/or the firewall to no longer accept connections from hosts where unauthorized access attempts came from.

Figures 1a and 2 present the trace used to count successful accesses to the web server. Figure 4 shows how to group several messages into a single transition. The traces used to count other reply codes are similar. To count unauthorized access attempts, for instance, one must define a trace to monitor the occurrence of the GET primitive followed by an HTTP/1.1 401 response. By monitoring several reply codes during regular polling times, the MLMs can generate detailed reports. 


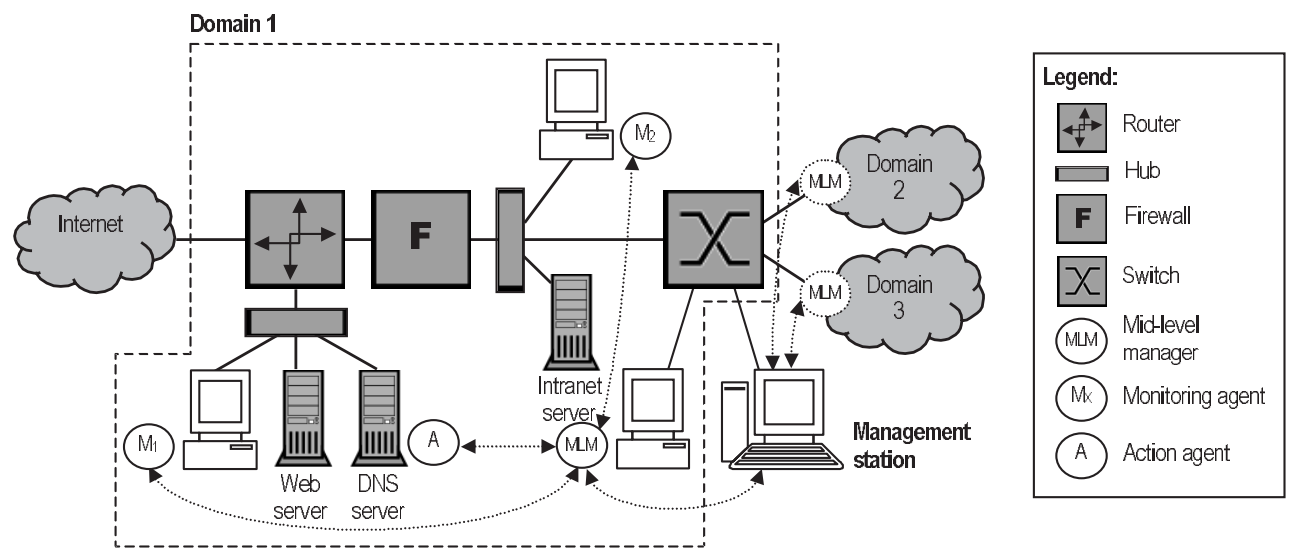

Figure 8: A network and some services

The monitoring of accesses to the web server located on the external network is done by programming the monitoring agent $M_{1}$, while the web server responsible for the Intranet is monitored through the monitoring agent $M_{2}$. Each monitoring process makes part of distinct management tasks, even though they run similar procedures.

\subsection{Security Management of DNS and Web Servers}

The DNS and web servers located on the external network may be vulnerable to malicious exploits coming from the Internet. The security monitoring of these services consists in monitoring the hosts where they are located and check whether they are victims of port scans, denial of service attacks, among other exploits that can be detected passively.

A port scan consists of sending packets to a range of ports of a host to know which TCP and UDP services are available. When using TCP, if the host does not have a service listening in a determined port, it will send back a TCP packet with the RST bit on in response to the connection attempt. Figure $9 \mathrm{a}$ and $\mathrm{b}$ presents this trace.

The mid-level manager programs the monitoring agent $M_{1}$ to start monitoring the trace. Furthermore, it will periodically poll the extended RMON2 MIB where the monitoring results are stored (see table 1). If during a polling interval the number of occurrences of the trace is higher than a determined value, defined by the manager, the script will generate a notification to the central management station.

A similar procedure is done when one of the stations suffers from an attack known as SYN Flood. This attack consists of sending a huge number of connection setup packets (TCP packet with the SYN flag on) with a fake source address to a target host. This fake address must be unreachable or non-existent (usually a reserved value). When the target host receives these SYN packets, it creates a new entry on its connection table and sends back a SYN/ACK packet to the possible client. After sending the reply packet, the target host waits for an acknowledge from the client to establish the connection. As the source address is fake, the server will wait a long time for this reply. In a given time, the connection queue of the server will be full and all new connection requests will be discarded, creating a denial of service. This state will last until the entries on the connection table start to timeout.
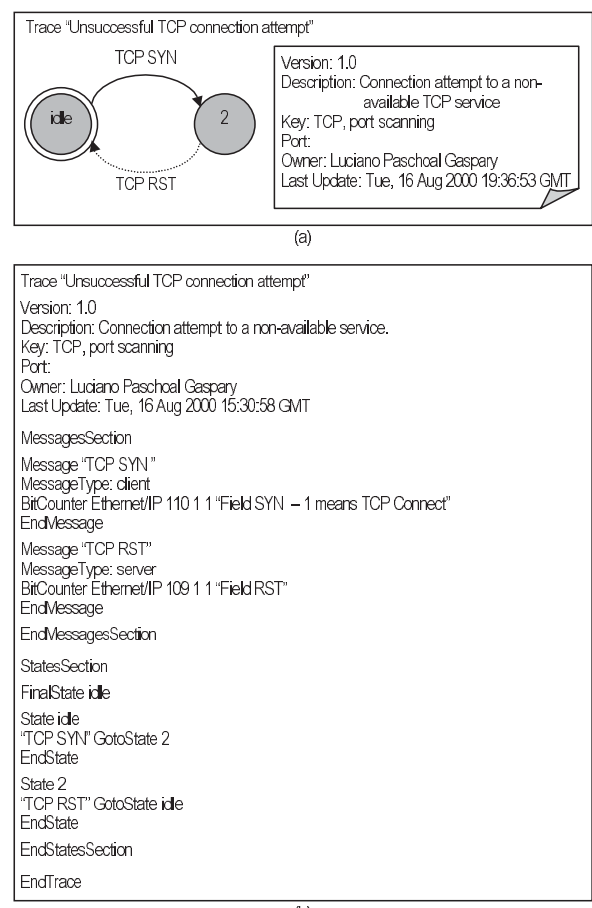

(b)

Figure 9: Trace to detect port scanning 
The identification of this attack is done by the trace shown in figure 10. Unlike other examples presented, this attack is identified by observing unsuccessful occurrences of the trace. This information is stored at the APM MIB, as shown in table 2.

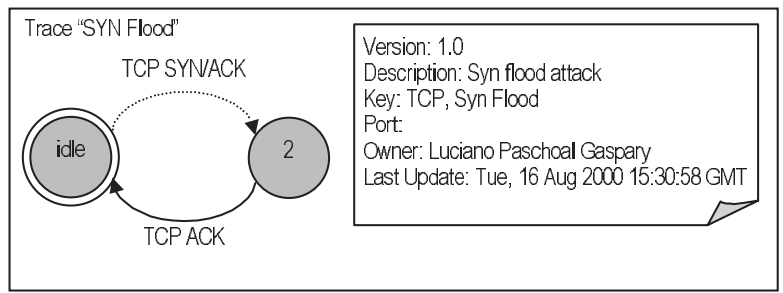

Figure 10: The SYN Flood attack

\section{Conclusions and Future Work}

This paper presented an architecture for distributed management of high-layer protocols and network services based on the use of programmable agents. Motivated by the increasing demand by organizations that need to manage high-layer protocols and their critical applications, this work proposed a flexible architecture that is able to keep up with the fast proliferation of protocols and network applications (that must be managed). Based on the IETF SNMP standard, this architecture does not require major changes in existing management systems (which took years to consolidate).

The proposal of the PTSL language is one of the most important contributions of this work. All approaches discussed and listed in Section 2 are limited to the accounting of sent/received packets between pairs of peers, classifying them based on protocols [2] or flows [1, 5]. In these approaches, the manager has access to information limited to the style "host A sent $n$ octets/packets to host B", with filters to some well-known protocols (e.g. HTTP and SMTP) or packets with specific header fields. The innovations aggregated with PTSL increase the granularity in which protocols are monitored, enabling the analysis of the behavior of a protocol or just part of a protocol by introducing the representation of desired traces. This provides the network manager with more accurate information, which will help him to deploy fault, configuration, accounting, performance and security management to high-layer network protocols and services. Using the previous example, the language allows the accounting of successful, unsuccessful and unauthorized HTTP accesses, as well as many other possible HTTP behaviors. The PTSL power of expression is another strong point. While many approaches allow the selection of packets based on a few predetermined header fields only up to the transport layer
[5], PTSL goes further, allowing the use of filters based on any protocol, all the way up to the application layer.

Integrated management is an inherent characteristic of the architecture. Instead of using specific tools to monitor individual protocols and services (e.g. web, video-on-demand and email), one can use the Trace architecture to monitor such protocols and services through a unified framework. By delegating the functionality of these tools to distributed management stations, our approach burdens off the workload on the hosts where these services are installed.

One positive aspect of the Trace architecture is the possibility of making effective management of high-layer network protocols and services by integrating the PTSL language with programmable monitoring agents and by associating the occurrence of specific traces to dynamically programmable actions, enabling the automation of a set of management procedures. The proposed architecture is not limited to monitoring, to the contrary, it provides a more complete and broader solution that includes the execution of actions, enabling both reactive and proactive management.

Another positive aspect of the architecture is a significant increase of scalability in relation to the traditional SNMP management paradigm, since it can delegate management tasks, previously processed only at the centralized management station, to MLMs. The robustness aggregated to the management tasks also represents an important contribution. The architecture allows the delegation of management functions to MLMs that are closer to the monitored agents; if the connection is lost between the centralized management station and the MLM, these management tasks will still be able to run. The delegation is not only about tasks, but it will also delegate CPU cycles and will keep polling as close as possible to the management targets.

However, this distributed architecture demands more work to be controlled. The component management becomes a quite complex task. Included in the component management are the distribution and update of scripts, the retrieval and correlation of results. One of the proposed future works is the creation of mechanisms that provide an even more transparent use of the architecture.

Regarding the implementation of the architecture it is important to say that the developed prototype is being improved to offer the transparency just mentioned. The network management environment interface, which was not a priority until now, is being redesigned. The procedures executed by the MLMs are being encapsulated into a Tcl package, so that the manager will not need wizards any- 
more, giving more power to specifications. Performance tests were not carried out yet, but Schoenwaelder presents good results in [11], where the Jasmin implementation was evaluated.

\section{References}

[1] L. Deri and S. Suin, "Ntop: Beyond Ping and Traceroute" Proc. 10th IFIP/IEEE Workshop on Distributed Systems: Operations and Management, Zurich, Oct. 1999, pp. 271-283, Springer Verlag.

[2] S. Waldbusser, "Remote Network Monitoring Management Information Base Version 2 using SMIv2", RFC 2021, INS, Jan. 1997.

[3] G. Malan and F. Jahanian, "An Extensible Probe Architecture for Network Protocol Performance Measurement", in Proc. of SIGCOMM, Vancouver, Sep. 1998.

[4] N. Brownlee, C. Mills, and G. Ruth, "Traffic Flow Measurement: Architecture", RFC 2722, The University of Auckland, GTE Laboratories Inc., GTE Internetworking, Oct. 1999.

[5] N. Brownlee, NeTraMet, http://www.auckland.ac.nz/net/Internet/rtfm/.

[6] N. Brownlee, "Traffic Flow Measurement: Meter MIB", RFC 2720, The University of Auckland, Oct. 1999.

[7] N. Brownlee, "SRL: A Language for Describing Traffic Flows and Specifying Actions for Flow Groups", RFC 2723, The University of Auckland, Oct. 1999.

[8] C. Cook et al., An Introduction to Tivoli Enterprise, First edition, International Technical Support Organization, 1999, http://www.redbooks.ibm.com.

[9] L. P. Gaspary and L. R. Tarouco, "Characterization and Measurements of Enterprise Network Traffic with RMON2", Proc. 10th IFIP/IEEE Workshop on Distributed Systems: Operations and Management, Zurich, Oct. 1999, pp. 229-242, Springer Verlag.

[10] S. Waldbusser, "Application Performance Measurement MIB”, Internet Draft, Jul. 2001.

[11] J. Schoenwaelder, J. Quittek, and C. Kappler, "Building Distributed Management Applications with the IETF Script MIB", IEEE Journal on Selected Areas in Communications, vol. 18, no. 5, pp. 702-714, 2000.

[12] D. Levi and J. Schoenwaelder, "Definitions of Managed Objects for the Delegation of Management Scripts", Internet Draft, Nortel Networks, TU Braunschweig, June 2001.
[13] L. P. Gaspary, L. F. Balbinot, R. Storch, F. Wendt, and L. R. Tarouco, "Towards a Programmable Agent-based Architecture for Enterprise Application and Service Management", in Proc. First IEEE/IEC Enterprise Networking Applications and Services Conference, Atlanta, June 2001, Piscataway, USA: IEEE Operations Center, 2001. p. 39-46.

[14] TU Braunschweig, NEC Cl\&C Research Laboratories, Jasmin - A Script MIB Implementation, 1999, http://www.ibr.cu.tu-bs. de/projects/jasmin.

[15] D. Levi, P. Meyer, and B. Stewart, "SNMP Applications", RFC 2573, SNMP Research Inc., Secure Computing Corporation, Cisco Systems, Apr. 1999.

[16] R. Fielding, J. Gettys, J. Mogul, H. Frystyk, T. BernersLee, "Hypertext Transfer Protocol - HTTP/1.1", RFC 2068, UC Irvine, DEC, MIT/LCS, Jan. 1997. 